\title{
APPLICATION OF CFD TO MODELING OF SQUEEZE MODE MAGNETORHEOLOGICAL DAMPERS
}

\author{
Janusz GOLDASZ ${ }^{* * *}$, Bogdan SAPIŃSKI"** \\ *BWI Group, Technical Center Kraków, ul. Podgórki Tynieckie 2, 30-399 Cracow, Poland \\ "Department of Automation and Information Technology, Cracow University of Technology, ul. Warszawska 24, 31-155 Cracow, Poland \\ ${ }^{* * *}$ Department of Process Control, AGH University of Science and Technology, al. Mickiewicza 30-059 Cracow, olnd \\ jgoldasz@pk.edu.pl, deep@agh.edu.pl
}

received 12 May 2015, revised 30 July 2015, accepted 7 September 2015

\begin{abstract}
The so-called squeeze flow involves a magnetorheological (MR) fluid sandwiched between two planar surfaces setting up a flow channel. The height of the channel varies according to a prescribed displacement or force profile. When exposed to a magnetic field of sufficient strength MR fluids develop a yield stress. In squeeze-mode devices the yield stress varies with both the magnetic field magnitude and the channel height. In this paper an unsteady flow model of an MR fluid in squeeze mode is proposed. The model is developed in Ansys Fluent R16. The MR material flow model is based on the apparent viscosity approach. In order to investigate the material's behaviour the authors prepared a model of an idealized squeeze-mode damper in which the fluid flow is enforced by varying the height of the channel. Using mesh animation, the model plate is excited, and as the mesh moves, the fluid is squeezed out of the gap. In the simulations the model is subjected to a range of displacement inputs of frequencies from $10 \mathrm{to} 20 \mathrm{~Hz}$, and local yield stress levels up to $30 \mathrm{kPa}$. The results are presented in the form of time histories of the normal force on the squeezing plate and loops of force vs. displacement (velocity).
\end{abstract}

Key words: Magnetorheological Fluid, Damper, Squeeze-Mode, Computational Fluid Dynamics, Mesh Animation

\section{INTRODUCTION}

The MR effect is manifested by changes in the material's apparent viscosity when exposed to magnetic field. This feature has made it more than welcome in semi-active control systems and automotive applications in particular. The MR material has been basis for developing hardware and applications also in the real world and not only the academia. By examining the manner in which the material operates it is now well understood the material may operate in any of the operation modes: flow-mode, shearmode, squeeze-mode (Jolly et al., 1996; Jolly and Carlson, 1996). Real-world examples of flow-mode hardware include automotive dampers and powertrain mounts. Rotary devices are controllable brakes and clutches. The squeeze-mode utilizes an MR fluid that is trapped in a flow channel whose height varies according to a prescribed displacement profile on either surface of the channel. The fluid is squeezed out of the gap simple by the plate motion. This particular operation mode may provide optimum performance when implemented in small-stroke dampers or vibration mounts. At the same time a successful application of squeeze-mode requires a mathematical model of the phenomena for fast sizing of prototypes at a design stage of the development process. Analytical models based on the classic Bingham approach (Gołdasz and Sapiński, 2015) are rather limited in scope, and the solutions have been made available in few idealized cases, e.g. simple geometry. CFD (Computational Fluid Dynamics) tools offer a modeling potential that can be realized within a reasonable time constraint, although full scale unsteady flow problems are a challenge, too.

CFD or finite-element studies on devices utilizing MR fluids are rare, e.g. (Case et al., 2013, Chen et al., 2007; Zheng et al., 2014), and on squeeze-mode devices even so (Gstottenbauer et al., 2008; Sapiński and Szczęch, 2013). Therefore, there is a need to address the problem of developing a model procedure for use with more complex case studies. In this study the authors apply an apparent viscosity model to a simple $2 \mathrm{D}$ axi-symmetric model of an idealized squeeze-mode damper geometry. The unsteady behavior of the incompressible fluid in squeeze flow is then studied using mesh animation techniques. The calculations are performed for a material in which the magnetic field-induced yield stress is allowed to vary in terms of the flow channel height

\section{MODELING}

In this section the authors was presented Navier-Stokes equation and the apparent viscosity model. Additionaly, the geometry of the damper, yield stress vs. gap height characteristics were highlighted.

\subsection{Theory}

The behaviour of MR fluids in flow can be studied using wellknown Navier-Stokes (N-S) equations. Their tensor form is (Tannehi et al., 1996)

$\rho \frac{\partial u_{i}}{\partial t}+\rho u_{j} \frac{\partial u_{i}}{\partial x_{j}}=-\frac{\partial p}{\partial x_{i}}+\frac{\partial \tau_{i, j}}{\partial x_{j}}$,

where: $\tau$ - shear stress, $\rho$ - density, $u$ - velocity, $p$ - pressure, and $x_{i}$ is the independent variable $(i=1,2,3)$. In N-S equations flow problems concerning specific materials are handled by the sheear stress term. In the case of MR fluids, using the Bingham model and defining the apparent viscosity as the ratio of the fluid's yield stress over the local shear rate the following expression is 
$\mu_{\text {app }}=\mu+\frac{\tau_{0}}{\left|\frac{\partial u_{i}}{\partial x_{j}}\right|}$

and the yield stress term

$\tau_{i, j}=\mu_{a p p} \frac{\partial u_{i}}{\partial x_{j}}$

where: $\tau_{0}$ - yield stress, $\mu_{a p p}$ - apparent viscosity, $\mu$ - base viscosity. The yield stress is allowed to vary in terms of space and magnitude. The model in Equation (2) has a discontinuity when the shear rate is zero which makes flow problems impossible to solve in these conditions. Solutions (Chen et al., 2007) suggest imposing an upper bound on the apparent viscosity term (2) when shear rate approaches zero. In the present model the upper bound is chosen to be 1000 times the base viscosity of the fluid. In Ansys Fluent this approach can be programmed into an userdefined function.

\subsection{Analytical solution}

Sproston et al. (1994) and Zhang et al. (2011) obtained an analytical solution for ER/MR fluids in squeeze-flow. In this paper the expression obtained by Zhang is further utilized in subsequent calculations. It is an extension of Stefan model (de Vincente et al., 2011; Esmonde et al., 2009; Farjoud et al., 2011) incl. the effects of viscosity, plasticity, and inertia (acceleration)

$F_{M R}=F_{v}+F_{\tau}-F_{i}$

and

$F_{v}=3 \frac{\mu \pi R_{p}^{4}}{2\left(h_{0}+z\right)^{3}} v_{z}$

$F_{\tau}=3 \frac{\pi R_{p}^{3} \tau_{0}}{4\left(h_{0}+z\right)} \operatorname{sgn}\left(v_{z}\right)$,

$F_{i}=3 \frac{\pi R_{p}^{4} \rho a_{z}}{8\left(h_{0}+z\right)}$

Where: $R_{p}$ - plate radius, $h_{0}$ - initial gap, $a_{z}$ - acceleration, $F_{M R}$ - normal force, $F_{v}$ - viscous force, $F_{i}$ - inertial force, $F_{\tau}$ - force due to yield stress. In this study results obtained using the analytical expression given by Equation (4) are compared against CFD.

\subsection{Input data}

Consider the 2D axi-symmetric geometry in Fig. 1. The outer rectange denotes the fluid domain, and the inner wall boundary refers to a circular flat plate that is constrained to move vertically according to the prescribed displacement profile $h(t)$. The fluid region directly underneath the plate is characterized by a volume of non-zero yield stress, whereas zero yield stress in imposed in the other portion of the fluid. The outer diameter $D_{p}$ of the plate is $64 \mathrm{~mm}$, and the initial gap height $h_{0}=3.38 \mathrm{~mm}$. The CFD model is setup with wall boundary conditions on the outer and the inner boundary. Mesh is animated on the inner boundary in order to simulate flow conditions of a fluid subjected to external kinetic (as well as magnetic) excitation inputs.

In the simulated study the authors assumed fluid properties similar to the BASF's 4035 MR material with $26 \%$ Fe vol (Kieburg, 2010). The density of the fluid is $2.6 \mathrm{~g} / \mathrm{cc}$, and the base viscosity $\mu=50 \mathrm{cP}$

The region of the fluid underneath the plate is assumed to be characterized by a magnetic field supplied from a virtual core assembly with a coil of $N=150$ wire turns. With the current range from 0 to $3 \mathrm{~A}$, the fluid's yield stress vs. height characteristics can be estimated in Fig. 2. The arrows in the figure indicate the operating range of this virtual device; the minimum gap height was $1.94 \mathrm{~mm}$, and the maximum height was $4.82 \mathrm{~mm}$.

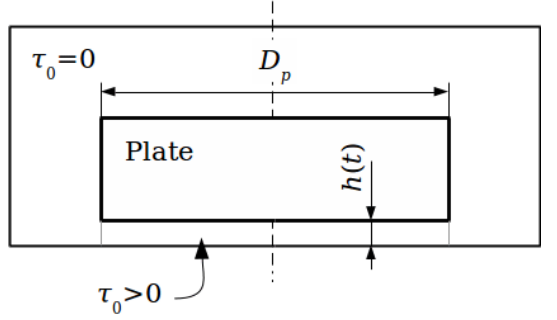

Fig. 1. CFD model schematic layout

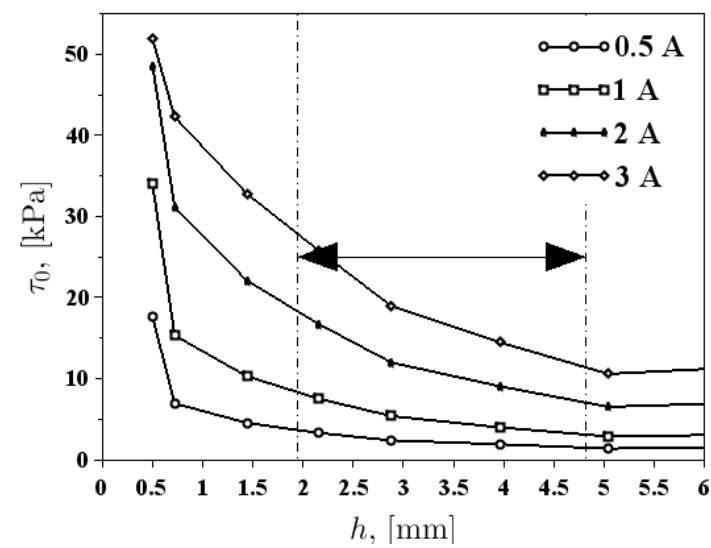

Fig. 2. Calculated yield stress vs. channel height, $\tau_{0}(h)$

To summarize, the series of simulated experiments incorporated two stages. The first one assumed constant yield stress of the fluid (regardless of the channel height), whereas as the second part utilized the variable yield stress model according to Fig. 2. The external excitation was in the form of sinusoidal displacement input applied to the inner boundary (plate). Given the initial gap size the gap height varied according to $h(t)=h_{0}+$ $z(t)=h_{0}+\cos (2 \pi f t+\pi / 2)$, where $z$ - plate displacement, $z_{m}$ - displacement amplitude, $f$ - frequency. The maximum aplitude of the displacement input was $1.44 \mathrm{~mm}$, and the frequency range was from $10 \mathrm{~Hz}$ to $20 \mathrm{~Hz}$, thus translating into peak velocities of the plate to be appr. $200 \mathrm{~mm} / \mathrm{s}$. In the CFD model the force is assumed to be positive in compression (downward motion).

\subsection{Results}

The section contains simulation results by means of Ansys Fluent R16. Unsteady flow equations were handled at the constant time step of $0.04 \mathrm{~ms}$. The data are presented in the form of plots of force vs. displacement (velocity) in Figs. 3-11.

To provide force estimate, pressure distribution was integrated across the entire surface area below the plate. 
a)

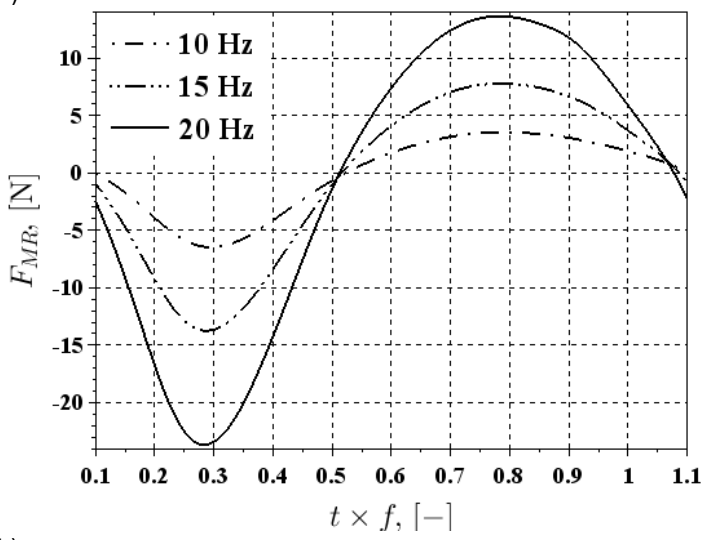

b)

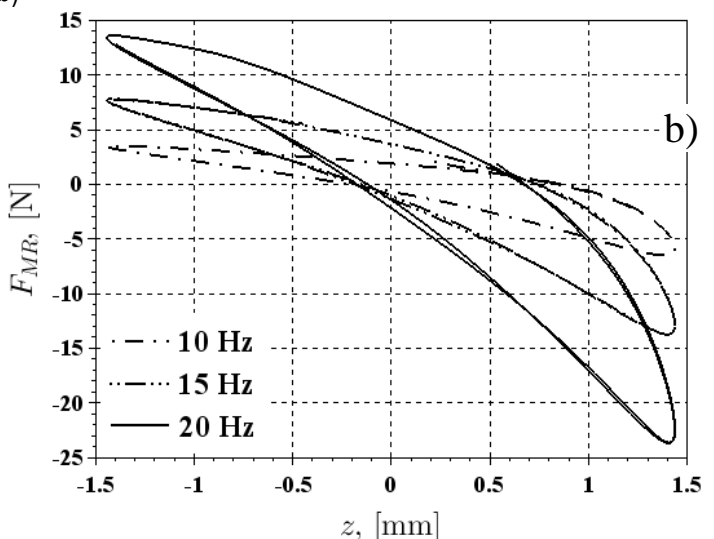

c)

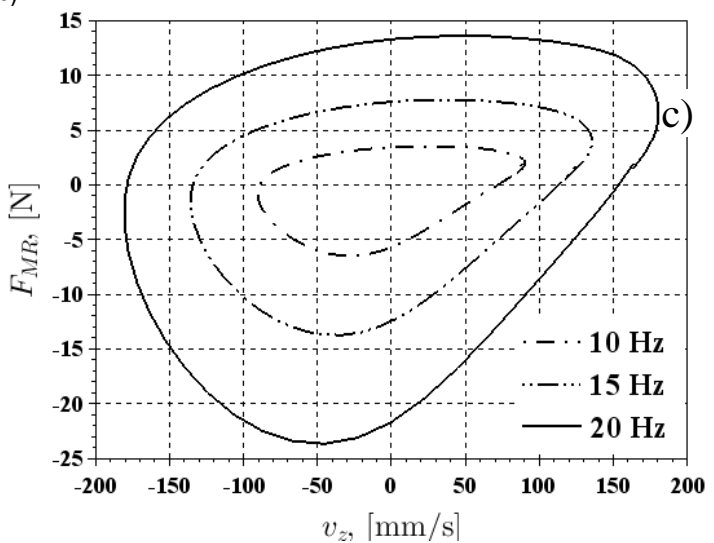

Fig. 3. Non-energized condition: $\tau_{0}=0 \mathrm{kPa}, f=\{10,15,20 \mathrm{~Hz}\}$,

a) $F_{M R}$ vs. $t$, b) $F_{M R}$ vs. $z$, c) $F_{M R}$ vs. $v_{z}$

a)

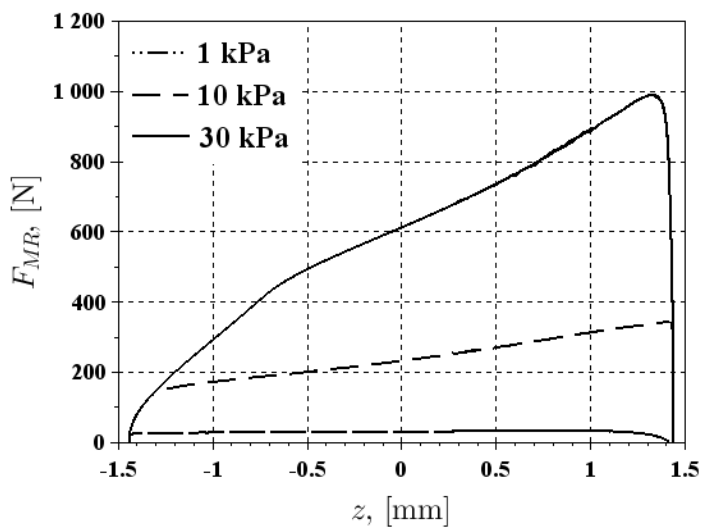

Fig. 5. Constant yield stress: $f=15 \mathrm{~Hz}$, a) $F_{M R}$ vs. $z$, b) $F_{M R}$ vs. $v_{z}$
Specifically, constant yield stress scenarios are revealed in Figs. 3-6. The effect of frequency can be studied by considering the results in Figs. 7-9. Next, a comparison of CFD results against the analytical squeeze-flow model as in Equation (4) is given in Fig. 10. Finally, variable yield stress cases are illustrated in Fig. 11. With the exception of Fig. 3. all illustrations show only the positive force output. Tensile forces were beyond the scope of this study. In Fig. 3a the simulation time $t$ is scaled by the input frequency $f$ to enable a direct comparison of the obtained results regardless of the kinetic excitation conditions. Fig. 3 reveals small yet noticeable effects of inertia manifested by a rotation of the force vs. displacement ellipse in Fig. 3b and the increase in the force vs. velocity hysteresis as in Fig. 3c.

a)

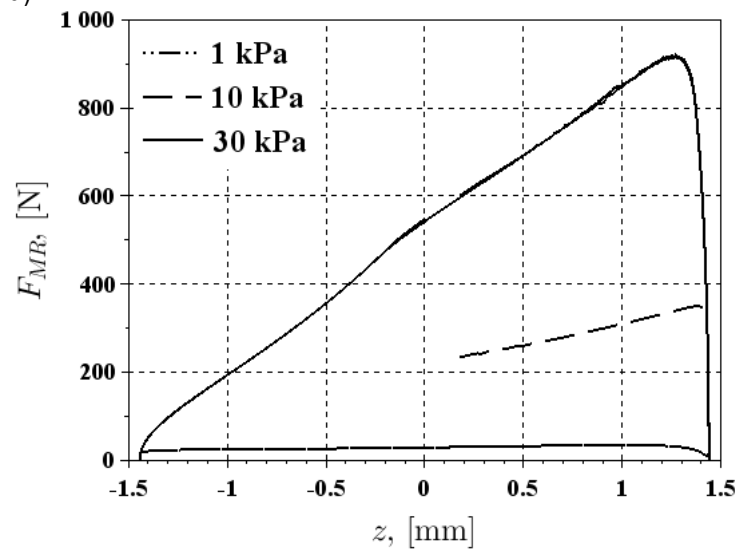

b)

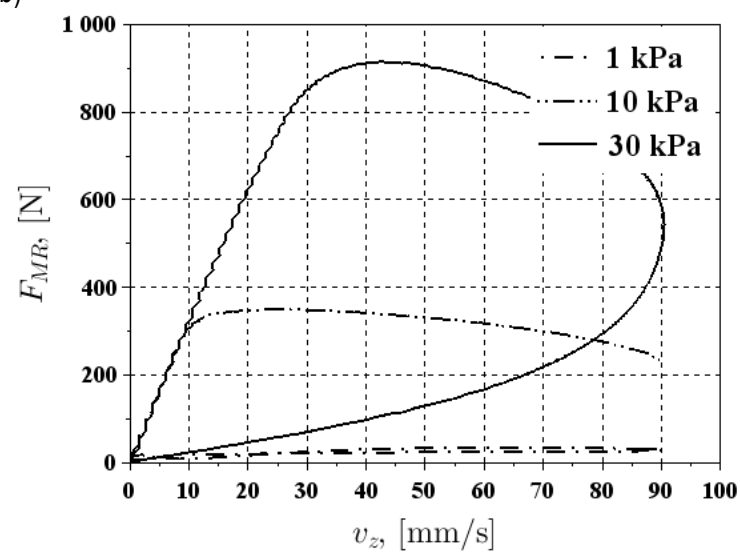

Fig. 4. Constant yield stress: $f=10 \mathrm{~Hz}$, a) $F_{M R}$ vs. $z$, b) $F_{M R}$ vs. $v_{z}$

b)

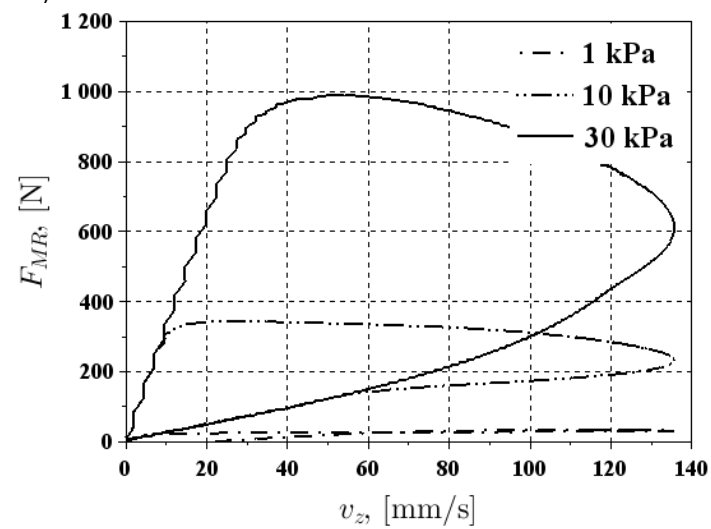


Constant non-zero yield stress results in Figs. 4, 5 and 6 were performed for three frequencies $(10,15,20) \mathrm{Hz}$ and three distinct levels of the yield stress $(1,10,30) \mathrm{kPa}$, respectively. Specifically, $20 \mathrm{~Hz}$ data in Fig. 6 reveal an appr. $100 \mathrm{~N}$ increase in the force due to higher shear rates. The $30 \mathrm{kPa}$ curve in Fig. 6 also shows a dual rate behavior that is almost absent in the corresponding data set in Fig. 4. The dual-rate feature is representative of the biviscous implementation of the apparent viscosity model in Fluent. Effectively, the model response in low frequency and high yield stress scenarios may be underestimated The effect is yet to be studied in detail, however, the initial observation is consistent with

a)

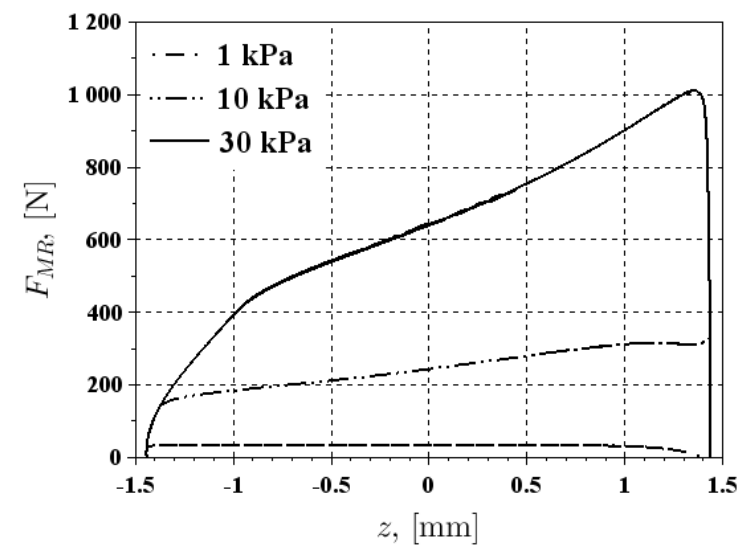

Fig. 6. Constant yield stress: $f=20 \mathrm{~Hz}$, a) $F_{M R}$ vs. $z$, b) $F_{M R}$ vs. $v_{z}$

a)

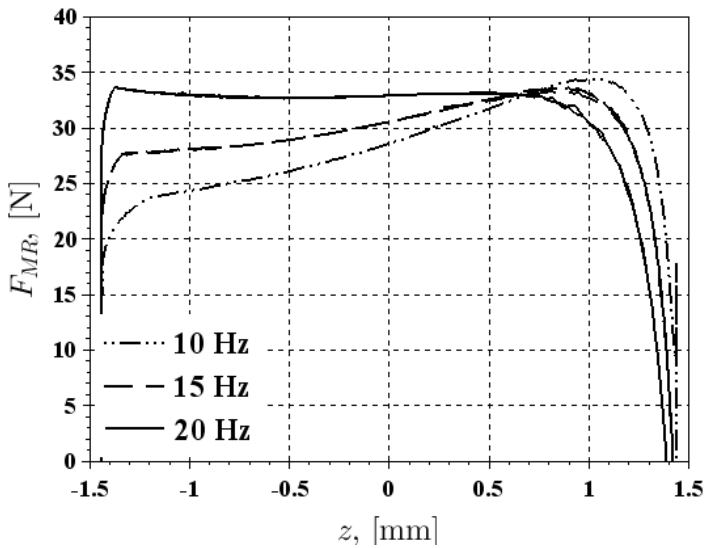

Fig. 7. Effect of frequency: $\tau_{0}=1 \mathrm{kPa}$ a) $F_{M R}$ vs. $z$, b) $F_{M R}$ vs. $v_{z}$

a)

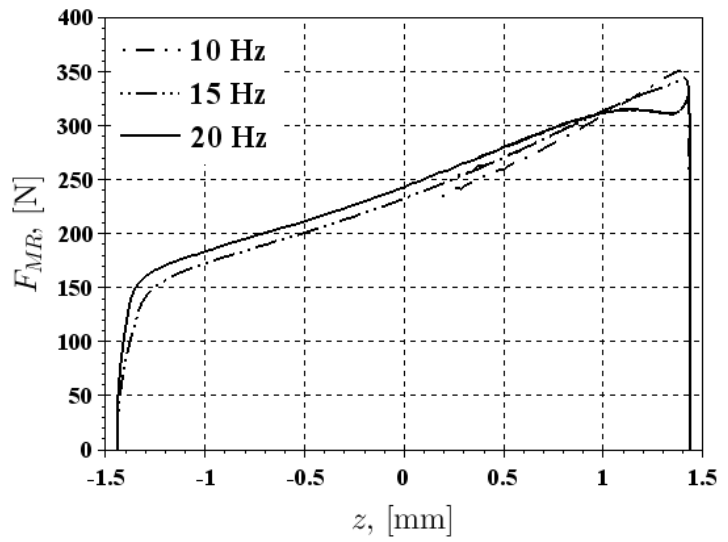

Fig. 8. Effect of frequency: $\tau_{0}=10 \mathrm{kPa}$, a) $F_{M R}$ vs. $z$, b) $F_{M R}$ vs. $v_{z}$ other data obtained using the same apparent viscosity model (Farjoud et al., 2011). In all cases the force shows a strong dependence on piston position. That is evident when observing a large hysteresis in force vs. velocity loops in Figs. 4-6. For comparison in Figs. 7-9 the influence of frequency (and velocity) can be observed and analyzed. Specifically, Fig. 7 reveals the model output at the constant yield stress of $1 \mathrm{kPa}$ and the three frequencies, respectively. Fig. 8 illustrates the $10 \mathrm{kPa}$ case, and Fig. 9 illustrates the behavior of the fluid whose yield stress was $30 \mathrm{kPa}$.

b)

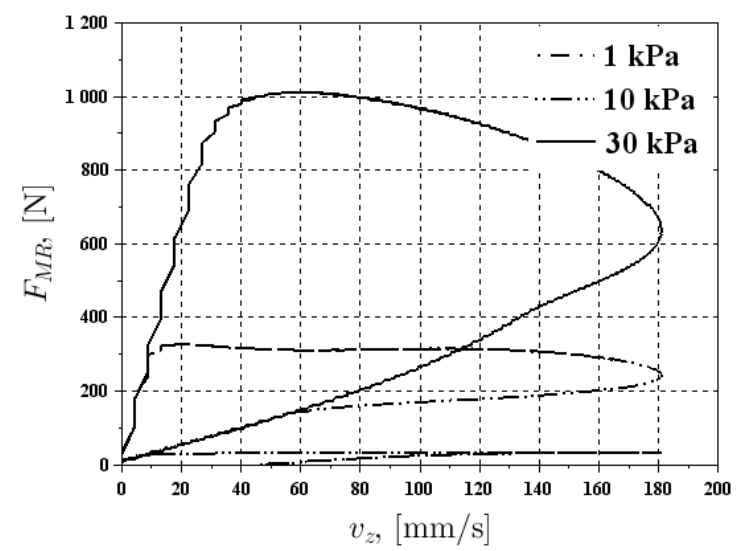

b)

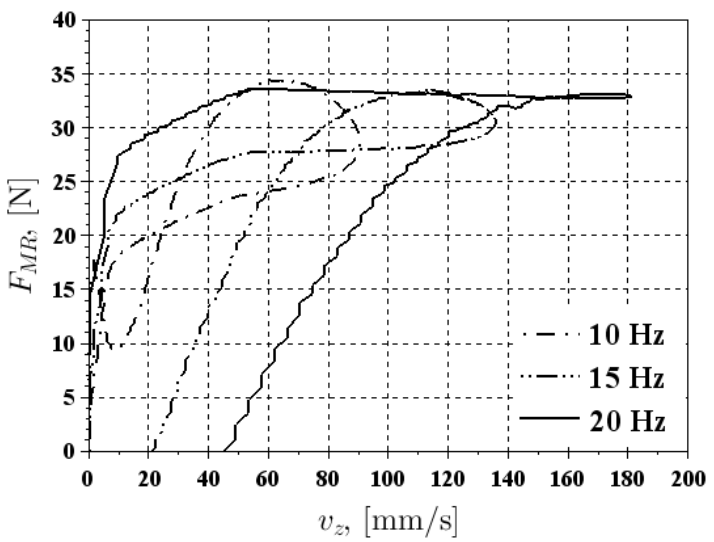

b)

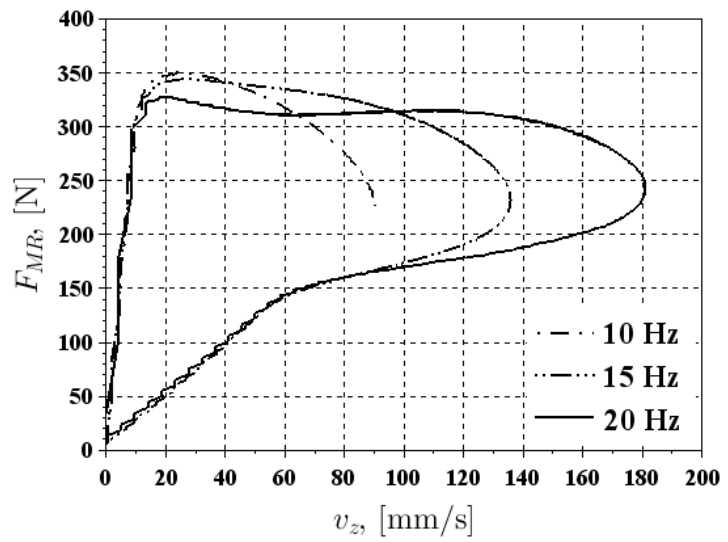


a)

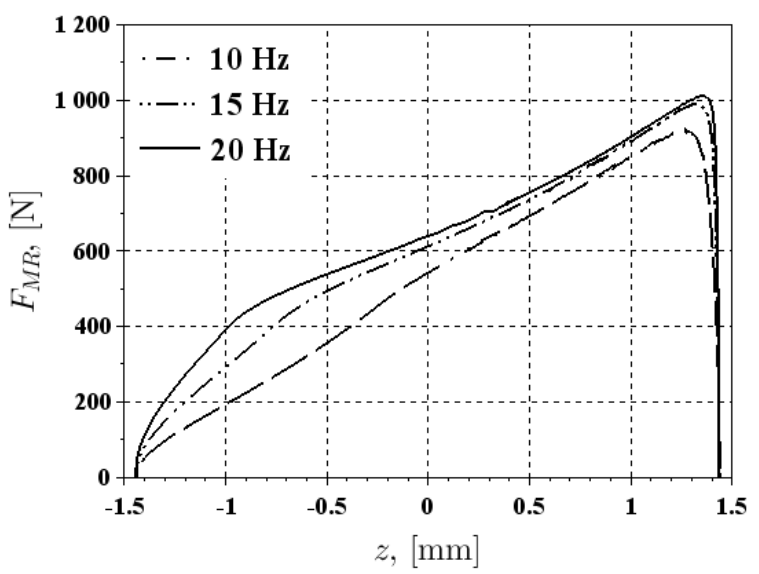

Fig. 9. Effect of frequency: $\tau_{0}=30 \mathrm{kPa}$, a) $F_{M R}$ vs. $z$, b) $F_{M R}$ vs. $v_{z}$

a)

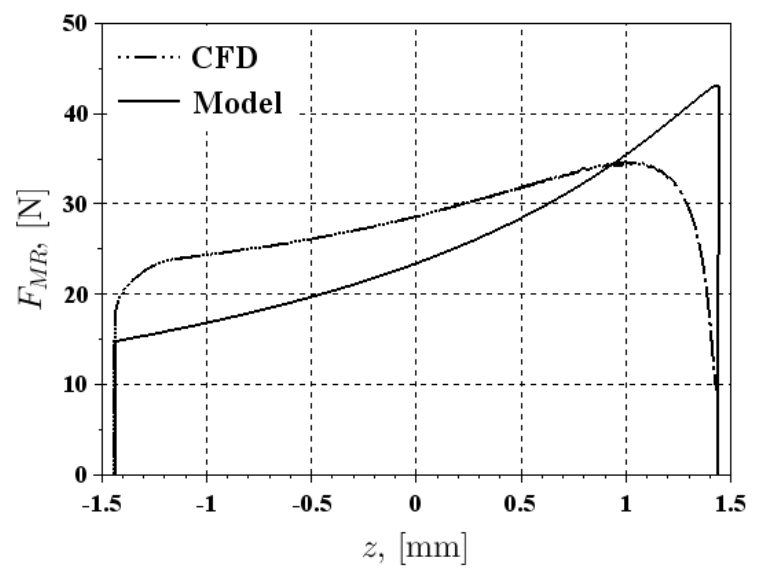

b)

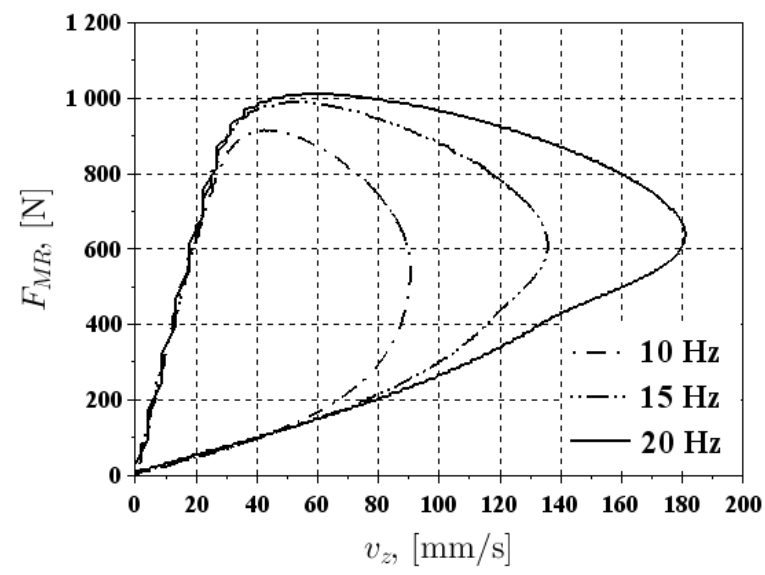

b)

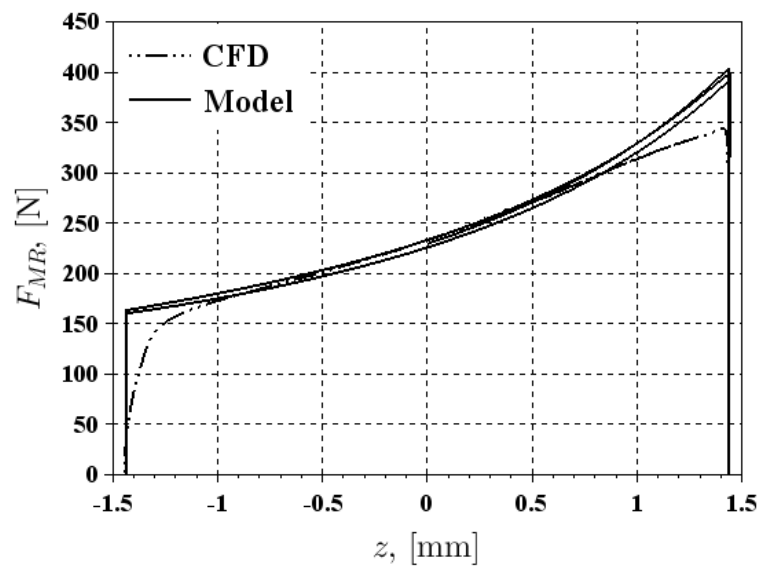

Fig. 10. Comparison: analytical model vs. CFD results, a) $\left.f=10 \mathrm{~Hz}, \tau_{0}=1 \mathrm{kPa}, \mathrm{b}\right) f=15 \mathrm{~Hz}, \tau_{0}=10 \mathrm{kPa}$

a)

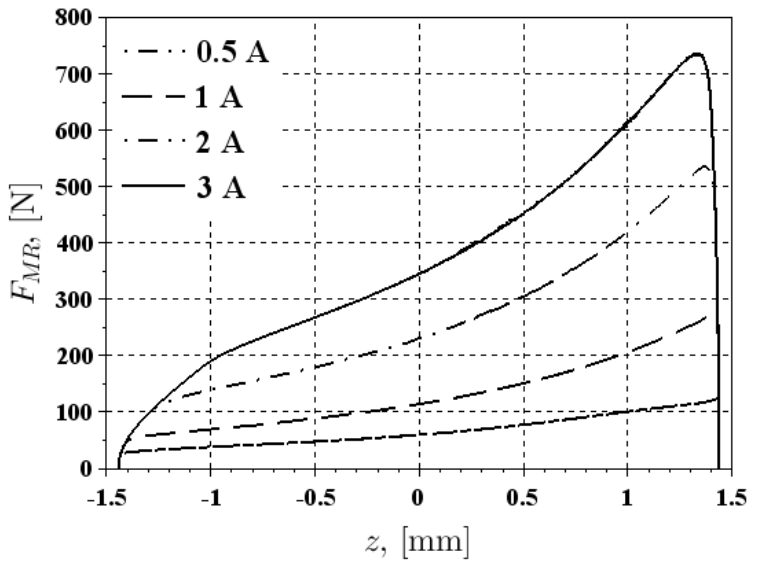

b)

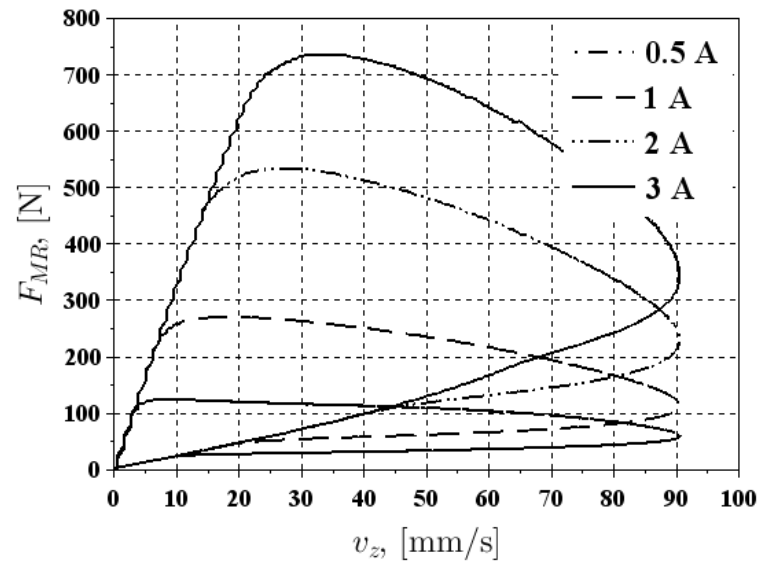

Fig. 11. Variable yield stress, $\tau_{0}=$ var, $f=10 \mathrm{~Hz}$, a) $F_{M R}$ vs. $z$, b) $F_{M R}$ vs. $v_{z}$

To validate the CFD model the obtained results were compared against the analytical expression given by Equation (4). The results are revealed in Fig. 10. In general, the illustrations show good agreement of the CFD output against the analytical theory. Discrepancies are at the extreme positions of the displacement range. The behavior is due to the bi-viscous character of the CFD solution, and is yet to be confirmed when analyzing the model performance against real data in near future. Finally, variable yield stress scenarios were considered in which the yield stress $\mathrm{T} 0$ was allows to vary in terms of the control gap height and current supplied by a virtual coil. The current range was from $0.5 \mathrm{~A}$ to $3 \mathrm{~A}$. The output is presented in Fig. 11. The effect of yield stress increase can be clearly seen in the revealed data. In the data the increase in the $F_{\tau}$ component with plate-to-base distance is accompanied by the effect of augmenting the yield stress due to position at current level. 


\section{CONCLUSIONS}

This study concerned a CFD experiment involving an MR fluid subjected to compressive loads. In general, the model confirms the well-known fact that as the gap height decreases, compressive loads increase. Specifically, the authors developed a CFD model based on the apparent viscosity approach and tested its performance under constant yield stress conditions and variable yield stress conditions, respectively. A comparison of the CFD data against theory was made, too, in order to draw conclusions on the model limitations and operation range. It is clear from the data that the Fluent model underestimates the analytical solution at near-zero velocity points, however, good agreement is obtained in all presented cases. The results provide a reasonable base for future work with real devices and fluids.

\section{REFERENCES}

1. Case D., Taheri, B., Richer, E. (2013), Multiphysics modeling of magnetorheological dampers, The International Journal of Multiphysics, Vol. 7, No. 1, 61-76.

2. Chen S. M., Bullough W. A., Ellam D. J. (2007), Examination of through flow in a radial ESF clutch, Journal of Intelligent Material Systems and Structures, Vol. 12, 1175-1179.

3. de Vicente, Juan, et al. (2011), Squeeze flow magnetorheology, Journal of Rheology (1978-present), Vol. 55, No. 4, 753-779.

4. Esmonde, H., H. See, and M. V. Swain (2009), Modelling of ER squeeze films at low amplitude oscillations, Journal of NonNewtonian Fluid Mechanics, Vol. 161, No. 1, 101-108.

5. Farjoud, A., Ahmadian, M., Mahmoodi, N., Zhang, X., \& Craft, M. (2011), Nonlinear modeling and testing of magneto-rheological fluids in low shear rate squeezing flows, Smart Materials and Structures, Vol. 20, No. 8, 085013.

6. Gołdasz J., Sapiński B. (2015), Insight into magnetorheological shock absorbers, Springer Publishing, Heidelberg.
7. Gstottenbauer, N., Kainz, A., Manhartsgruber, B., Scheidl, R. (2008), Experimental and numerical studies of squeeze-mode behaviour of magnetic fluid, Proceedings of the Institution of Mechanical Engineers, Part C: Journal of Mechanical Engineering Science, Vol. 22, No. 12, 2395-2407.

8. Jolly M., Bender J. W., Carlson J. D. (1996), Properties and applications of magnetorheological fluids, Proceedings of the SPIE Conference of the International Society of Optical Engineers, Vol. 3327, 262-275.

9. Jolly M., Carlson J. D. (1996), Controllable squeeze-film damping using magnetorheological fluids, Proceedings of the $5^{\text {th }}$ International Conference on New Actuators, Bremen, 333-336.

10. Kieburg Ch. (2010), MR Fluid Basonetic 4035, BASF Technical Information.

11. Sapiński, B., Szczęch, M. (2013), CFD model of a magnetorheological fluid in squeeze mode, acta mechanica et automatica, Vol. 7 , No. 3, 180-183.

12. Sproston J. L., Rigby S. G., Wiliams E. W., Stanway R. (1994), A numerical simulation of electrorheological fluids in oscillatory compressive squeeze-flow, Journal of Physics D: Applied Physics, Vol. 2, No. 27, 338-340.

13. Tannehill J. C., Anderson D. A., Pletcher R. H. (1996), Computational fluid mechanics and heat transfer. Taylor and Francis, New York.

14. Zhang X. J., Farjoud A., Ahmadian M, Guo K. H., Craft M. (2011), Dynamic testing and modeling of an MR squeeze mount, Journal of Intelligent Material Systems and Structures, Vol. 22, No. 15, 17171728.

15. Zheng, J., Li, Z., Koo, J., Wang, J. (2014), Magnetic circuit design and multiphysics analysis of a novel MR damper for applications under high velocity. Advances in Mechanical Engineering, Vol. 2014, 402501.

The work has been accomplished within the scope of the research project No. PBS 1/A6/3/2012 sponsored by the National Center for Research and Development (Poland). 